\title{
Connecting the dots in magnetic reconnection
}

Four formation-flying spacecraft reveal the 3D electron physics of magnetic reconnection Andrew J. Coates, Mullard Space Science Laboratory, Department of Space and Climate Physics, University College London, London WC1E 6BT, UK. Email: a.coates@ucl.ac.uk INSIGHTS | PERSPECTIVES 3 JUNE 2016 SCIENCE sciencemag.org • VOL 352 ISSUE 62901176

That plasmas don't mix well is a key aspect of this magnetized, highly conducting fourth state of matter. Magnetized plasmas are ubiquitous in our solar system, in astrophysics, and in the lab. The same fundamental process is common to solar flares, coronal mass ejections, the solar wind, the magnetospheres of Earth and other planets, comet tails, magnetars, and tokamaks. We know that plasma regions with embedded magnetic fields actually do spontaneously mix via the process of magnetic reconnection, with explosive results-we see the effects of particle acceleration in the aurora and solar energetic particles. On page 1189 of this issue, Burch et al. (1) present results that help shed light on the process of magnetic reconnection. They have probed a magnetic reconnection site in Earth's magnetosphere using a constellation of four spacecraft in close proximity, with unprecedentedly fast electron measurements to reveal what triggers reconnection and the vital role that electrons play on the small scale.

Plasma is not just a superheated gas with fluid-like properties; additional interest and complexity comes from its constituents: charged particles, electrons, and ions. On large length scales, the gas can be approximated as a fluid but with additional collective effects associated with the charges. This is the realm of magnetohydrodynamics (MHD). For a plasma of infinite conductivity (ideal MHD), the magnetic field becomes "frozen in" to the plasma flow. In this approximation the plasma behaves like a magnetized gas, leading to separate regions of the plasma with different characteristics (2). On smaller length scales, the motions of individual particles become important. The mass dependence of particle gyration in a magnetic field means that ions have a much larger gyration radius than electrons. To understand all the physics, in particular the ability to mix plasma across fluid boundaries, we must zoom in from the large, fluidlike scale to the kinetic scale with ions, and ultimately, electrons.

Since reconnection was first proposed as the mechanism that allows the solar wind to pass Earth (3), it has been seen as a key process. Reconnection may happen when oppositely directed magnetic fields spontaneously connect, accelerating particles as part of the process. Reconnection occurs between solar wind and terrestrial magnetic field at the magnetopause, and between oppositely directed magnetic fields in Earth's magnetotail. A major focus of solar-terrestrial physics concerns the structure of the reconnection region, the details of how it is triggered, and how it leads to magnetic storms (4). But the fundamental question of how the reconnection process works has remained unresolved.

Early models for reconnection included resistive diffusion occurring over small distance scales $(5,6)$, but this cannot explain some of the fast reconnection rates observed in solar flares. Slow mode shocks were then proposed (7) to play a role in separating the inflow and outflow regions, providing faster rates, although some approximations such as anomalous resistivity were not appropriate. A fully kinetic description, involving both ion and electron 
diffusion regions, was found to be important in theory and simulations. At progressively smaller scales, the ions and electrons become demagnetized as diffusion becomes important and dissipation results.

Earth's magnetopause and tail provide excellent laboratories for studying reconnection. Space-based missions are able to measure not only magnetic and electron fields, but also particle flows, directions, and velocities (the velocity distribution function) for electrons and ions, and they can measure plasma waves, which are important in understanding the physics. In general, a charged particle flow is a current that shapes the magnetic field, and particle distribution functions may be unstable and drive instabilities that can shape and disrupt the flow (4).

With advances in computing power, we are now getting to the stage where enough particles can be followed for a full kinetic simulation of reconnection, and space missions are keeping pace. In the 1970s and 1980s, dual, widely spaced space missions made some progress, but starting with Cluster in 2000, more closely spaced multi-spacecraft missions have been able to probe reconnection regions near to Earth (8). Together with the increasing capability of plasma particle instruments, this has led to good advances in our understanding of reconnection at Earth, Mercury (9), and Saturn (10). But the Cluster particle measurements relied on spacecraft spin to view the whole sky, and the time resolution was limited to half a spin.

The key aspect of the Magnetospheric Multiscale (MMS) mission (see the figure) is to look at the physics of reconnection in detail, in the electron diffusion/dissipation region. This requires kilometer-scale separation, 30-ms resolution on 3D measurements of electrons, and measurements of the 3D electric field, as well as good magnetic field measurements. This required a leap in technology from using one or two particle sensors on earlier missions, to remove the dependence on spacecraft spin. Onboard each MMS spacecraft are four dual-electron sensors, making a total of eight sensors measuring electrons simultaneously.

Burch et al. report the first detailed kinetic electron physics associated with magnetic reconnection at Earth's magnetopause and compare the findings with state-of-theart simulations. The results include a precise measurement of the electric field associated with reconnection and the identification of current-carrying electrons that shape the structure and result in particle acceleration. Some of the results obtained from the data are beyond those available from simulations at present and will inspire new simulation analysis (for example, the formation of a nonpredicted crescent electron distribution along the parallel velocity direction, indicative of field line opening). The results should prove an important data set for researchers to better understand space, laboratory, and astrophysical plasmas. j

\section{REFERENCES}

1. J. L. Burch et al., Science 352, XXX (2016),

2. H.O. G. Alfven, IEEE Trans. Plasma Sci. 18, 5 (1990).

3. J. W. Dungey, Phys. Rev. Lett. 6, 47 (1961).

4. S. A. Fuselier, W. S. Lewis, Space Sci. Rev. 160, 95 (2011). 
5. P. A. Sweet, in IAU Symposium 6, Electromagnetic Phenomena in Cosmical Physics, B. Lehnert, Ed. (Kluwer, Dordrecht, Netherlands, 1958), p. 123.

6. E. N. Parker, J. Geophys. Res. 62, 509 (1957).

7. H. E. Petschek, in The Physics of Solar Flares, proceedings of the AAS-NASA Symposium, 28 to 30 October 1963 (Goddard Space Flight Center, Greenbelt, MD, 1964), p. 425.

8. G. Paschmann, M. Øieroset, T. Phan, Space Sci. Rev. 178, 385 (2013).

9. J. A. Slavin et al., Science 324, 606 (2009).

10. C. S. Arridge et al., Nat. Phys. 12, 268 (2016).

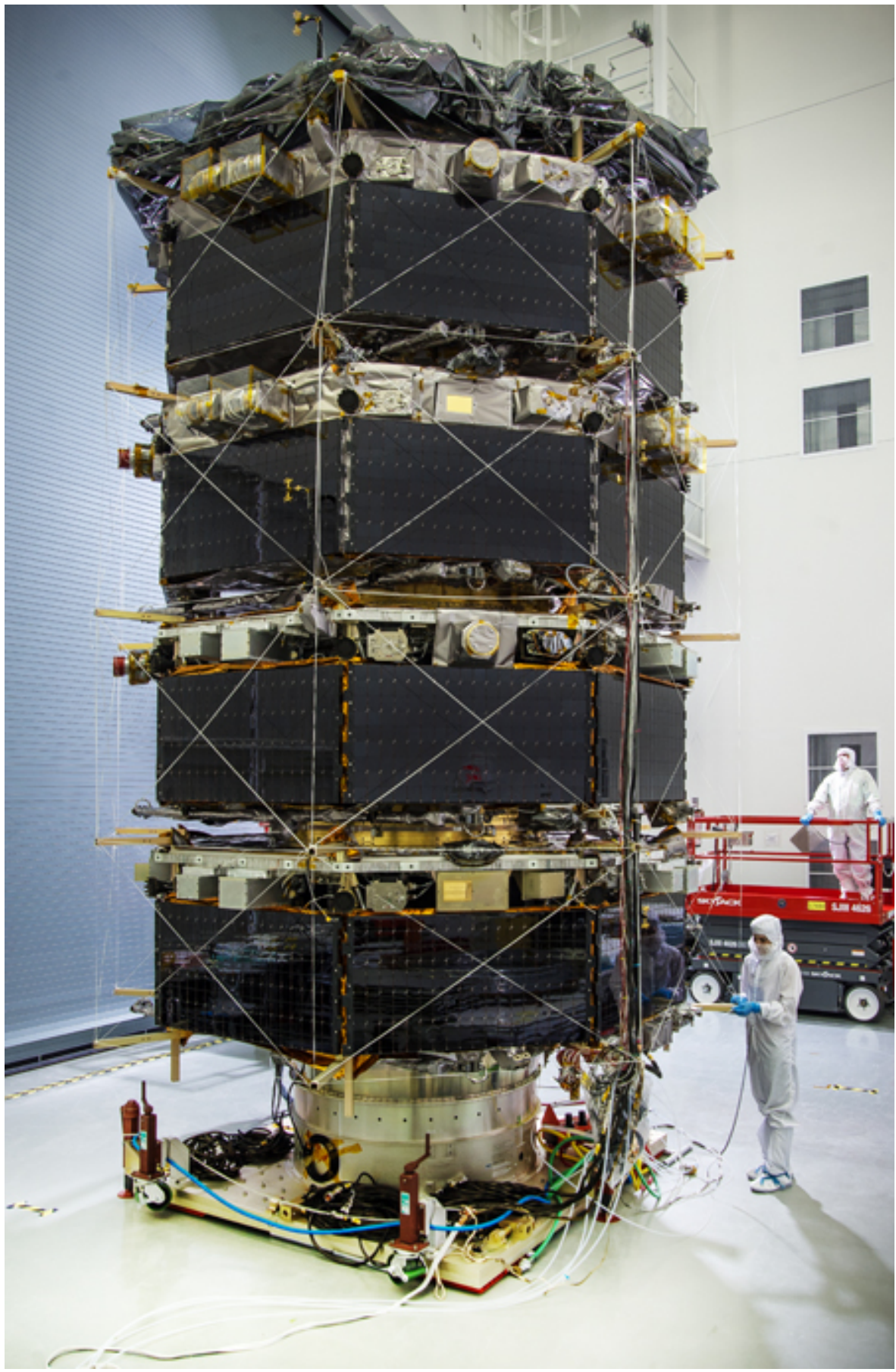

The four MMS spacecraft showing the multiple particle detectors and other instruments above the solar panels. 